Commun. Korean Math. Soc. 24 (2009), No. 3, pp. 451-458

DOI 10.4134/CKMS.2009.24.3.451

\title{
A NOTE ON THE SET OF ROOT CLASSES
}

\author{
Seoung Ho Lee
}

\begin{abstract}
The set of root classes plays a crucial role in the Nielsen root theory. Extending Brown et al.'s work on the set of root classes of iterates of maps, we rearrange it into the reduced orbit set and show that under suitable hypotheses, any reduced orbit has the full depth property as in the Nielsen type theory of periodic orbits.
\end{abstract}

\section{Introduction}

Let $f: X \rightarrow X$ be a selfmap of a path connected topological space and $a \in X$. A root of $f$ at $a$ is a point $x \in X$ that is a solution to the equation $f(x)=a$. Two root $x, y$ of $f$ at $a$ are in the same root class of $f$ if there exists a map $p:(I, 0,1) \rightarrow(X, x, y)$ such that $f \circ p \simeq a_{*}$, where $\circ$ denotes composition of functions, $\simeq$ denotes path homotopy and $a_{*}$ is the constant path at $a$. We write $\mathcal{R}(f)$ for the set of the root classes of $f$.

For the integer $n>0$, the iterates of a map $f: X \rightarrow X$ are defined by $f^{1}=f$ and $f^{n+1}=f \circ f^{n}$.

Let $X$ be a compact connected ANR and $f: X \rightarrow X$ be a map. The set of fixed point classes of $f$ will be denoted $\mathcal{F} P(f)$. In the Nielsen type theory of periodic orbits [2, Section III.3], fixed point classes of the iterate $f^{n}: X \rightarrow X$ will also be called $n$-periodic point classes of $f$. Then $f$ acts on the set $\mathcal{F} P\left(f^{n}\right)$ by $A_{f^{n}} \mapsto f\left(A_{f^{n}}\right)$. The $f$-orbit of a fixed point class $A_{f^{n}}$ is called an $n$-orbit class, denoted by $A_{f}^{(n)}$. The set of $n$-orbit classes is denoted by $\mathcal{O}^{(n)}(f)$. The length of the orbit $A_{f}^{(n)}$ is the smallest integer $\ell>0$ such that $A_{f^{n}}=f^{\ell}\left(A_{f^{n}}\right)$.

Let $a$ be the base point in $X$, and take a path $w$ from $a$ to $f(a)$ as the base path for $f$. The induced endomorphism $f_{w}: \pi_{1}(X, a) \rightarrow \pi_{1}(X, a)$ is defined by $f_{w}(\langle\gamma\rangle):=\left\langle w f(\gamma) w^{-1}\right\rangle$ for any loop $\gamma$ at $a$. It is well known that every fixed point class of $f$ is assigned a Reidemeister class in $\mathcal{R}\left(f_{w}\right)$, called its coordinate. We get an injection $\rho: \mathcal{F} P(f) \hookrightarrow \mathcal{R}\left(f_{w}\right)$, where $\mathcal{R}\left(f_{w}\right)$ is the Reidemeister set in $\pi_{1}(X, a)$, defined by $\rho\left(A_{f}\right):=\left[c f\left(c^{-1}\right) w^{-1}\right]_{f_{w}}$ for any path $c$ from $a$ to a

Received July 31, 2008; Revised June 4, 2009.

2000 Mathematics Subject Classification. Primary 55M20, 57N99.

Key words and phrases. roots of map iterates, reduced orbit sets, full depth property. 
point $x$ in $A_{f}$. Thus we also get an injection

$$
\rho: \mathcal{O}^{(n)}(f) \hookrightarrow \mathcal{R} O^{(n)}\left(f_{w}\right)
$$

where $\mathcal{R} O^{(n)}\left(f_{w}\right)$ is the Reidemeister orbit set in $\pi_{1}(X, a)$, by $\rho\left(A_{f}^{(n)}\right):=$ $\left[c f^{n}\left(c^{-1}\right) f^{n-1}\left(w^{-1}\right) \cdots f\left(w^{-1}\right) w^{-1}\right]_{f_{w}}^{(n)}$ for any path $c$ from $a$ to a point $x$ in $A_{f}^{(n)}$. If $\ell \mid n$ and an $\ell$-orbit class $B_{f}^{(\ell)}$ lies inside an $n$-orbit class $A_{f}^{(n)}$, then their coordinates are related by $\rho\left(A_{f}^{(n)}\right)=\iota_{\ell, n}\left(\rho\left(B_{f}^{(\ell)}\right)\right)$, where $\iota_{\ell, n}$ is the levelchange function defined by $\iota_{\ell, n}(\beta):=\beta f^{\ell}(\beta) f^{2 \ell}(\beta) \cdots f^{n-\ell}(\beta)$, hence $\rho\left(A_{f}^{(n)}\right)$ is reducible to level $\ell$. The depth of an $n$-orbit class $A_{f}^{(n)}$ is defined to be the depth of its coordinate $\rho\left(A_{f}^{(n)}\right)$, i.e., the lowest level $d=d\left(\rho\left(A_{f}^{(n)}\right)\right)$ to which $\rho\left(A_{f}^{(n)}\right)$ reduces is its depth. A Reidemeister orbit is said to have the full depth property if its depth equals its length, i.e., $d=\ell$ (see [3]).

In this paper, extending Brown et al.'s work (see [1]), we will study the solutions to the equation $f^{n}(x)=a$ for some $a \in X$ which are the roots of $f^{n}$ as in the Nielsen type theory above. The recurrence number $\rho=\rho(f, a)$ of $f$ at $a$ is the least positive integer $\rho$ such that $f^{\rho}(a)=a$. Suppose $\rho \leq n$. Let $R^{n}$ be a root class of $f^{n}$ and $x \in R^{n}$ be given. Then the $f^{\rho}$-image $f^{\rho}\left(R^{n}\right)$ is the unique root class of $f^{n}$ containing $f^{\rho}(x)$. Thus we can define the reduced $f^{\rho}$-orbit set of $f$ denoted by $\mathcal{R}_{\rho}^{(n)}(f)$ and extend to the reduced orbit set of the group homomorphism induced by $f$.

In the last section, we define the full depth property of a reduced $f^{\rho}$-orbit of a root class of $f^{n}$ and show that any reduced $f^{\rho}$-orbit has the full depth property under suitable hypotheses.

\section{Reduced orbit sets of root classes of iterated maps}

Let $f: X \rightarrow X$ be a selfmap of a path connected topological space, let $n$ be a positive integer, and let $a$ be a given point in $X$. We introduce the definition of the root class of the $n$th iterate of $f$ in [1] and extend its properties. Two roots $x, y$ of $f^{n}$ at $a$ are in the same root class of the $n$th iterate $f^{n}$ of $f$ if there exists a path $p:(I, 0,1) \rightarrow(X, x, y)$ such that $f^{n} \circ p \simeq a_{*}$, where $\circ$ denotes composition of functions, $\simeq$ denotes path homotopy and $a_{*}$ is the constant path at $a$. We denote $R^{n}$ for a root class of $f^{n}$ and write $\mathcal{R}\left(f^{n}\right)$ for the set of all root classes of $f^{n}$. The recurrence number $\rho=\rho(f, a)$ of $f$ at $a$ is the least positive integer $\rho$ such that $f^{\rho}(a)=a$. A root of $f^{n}$ is reducible if it is a root of $f^{m}$ for some $1 \leq m<n$, and irreducible otherwise. If $\rho=\infty$, then all roots of $f^{n}$ are irreducible.

Let $R^{n}$ be a root class of $f^{n}$ and $x \in R^{n}$ be given. Suppose $\rho \leq n$. Then the $f^{\rho}$-image $f^{\rho}\left(R^{n}\right)$ is the unique root class of $f^{n}$ containing $f^{\rho}(x)$. We observe that $f^{\rho}\left(R^{n}\right)$ is independent of the choice of $x$ because if $p:(I, 0,1) \rightarrow(X, x, y)$ is a path such that $f^{n} \circ p \simeq a_{*}$, then $f^{\rho}(p):(I, 0,1) \rightarrow\left(X, f^{\rho}(x), f^{\rho}(y)\right)$ and $f^{n}\left(f^{\rho}(p)\right)=f^{\rho}\left(f^{n}(p)\right) \simeq f^{\rho}\left(a_{*}\right)=a_{*}$. Moreover, we have the characterization 
of $f^{\rho}$-images of root classes of $f^{n}$. We denote the root class of $f^{n}$ containing the root $x$ of $f^{n}$ by $R_{x}^{n}$.

Lemma 2.1. Suppose $\rho \leq n$ and $R_{x}^{n} \in \mathcal{R}\left(f^{n}\right)$, then there exist two-type sequences of root classes of $f^{n}$ as follows:

(1): If $\rho \mid n$, then there exists a reduced sequence;

$$
R_{x}^{n}, f^{\rho}\left(R_{x}^{n}\right), \ldots,\left(f^{\rho}\right)^{n / \rho}\left(R_{x}^{n}\right)=R_{a}^{n}=\left(f^{\rho}\right)^{n / \rho+1}\left(R_{x}^{n}\right)=\cdots .
$$

(2): If $\rho \nmid n$, then there exists a reduced sequence;

$$
\begin{aligned}
& R_{x}^{n}, f^{\rho}\left(R_{x}^{n}\right), \ldots, f^{(m+1) \rho}\left(R_{x}^{n}\right)=R_{f^{\rho-r}(a)}^{n}=f^{(m+2) \rho}\left(R_{x}^{n}\right)=\cdots, \\
& \text { where } n=m \rho+r \text { and } 0<r<\rho \text { for some } m, r \in \mathbb{Z} .
\end{aligned}
$$

Proof. (1) If $\rho \mid n$, then there exists $m \in \mathbb{Z}$ such that $n=m \rho$, so

$$
f^{n}\left(R_{x}^{n}\right)=f^{m \rho}\left(R_{x}^{n}\right)=R_{a}^{n}=f^{(m+1) \rho}\left(R_{x}^{n}\right)=\cdots .
$$

(2) If $\rho \nmid n$, then we have

$$
f^{(m+1) \rho}(x)=f^{(\rho-r)+n}(x)=f^{\rho-r}(a) .
$$

Thus we get the conclusion.

Definition 2.2. For $\rho \leq n$, from Lemma 2.1, we note that any reduced sequences of root classes of $f^{n}$ are limited to either $R_{a}^{n}$ or $R_{f^{\rho-r}(a)}^{n}$ for some $r$. Thus the root classes $R_{a}^{n}$ and $R_{f^{\rho-r}(a)}^{n}$ will be called the center of the set $\mathcal{R}\left(f^{n}\right)$ with respect to the recurrence number.

Definition 2.3. For $\rho \leq n$. The reduced $f^{\rho}$-orbit of a root class $R^{n}$ of $f^{n}$ is the set

$$
R^{(n)}=\left\{R^{n}, f^{\rho}\left(R^{n}\right), f^{2 \rho}\left(R^{n}\right), \ldots, f^{m \rho}\left(R^{n}\right) \mid m \rho<n+\rho\right\} .
$$

We define the length $\ell\left(R^{(n)}\right)$ of the reduced $f^{\rho}$-orbit $R^{(n)}$ as $\ell\left(R^{(n)}\right)=\# R^{(n)}$ the cardinality of the set $R^{(n)}$. The set of all such reduced $f^{\rho}$-orbits will be called the reduced $f^{\rho}$-orbit set of $f$, denoted by $\mathcal{R}_{\rho}^{(n)}(f)$.

Remark 2.4. Note that any reduced $f^{\rho}$-orbits of root classes of $f^{n}$ contain the center and the length of the reduced $f^{\rho}$-orbit of the center is 1 .

Example 2.5. Let $S^{1}=\{z:|z|=1\}$ be the unit circle in the complex plane and let $f: S^{1} \rightarrow S^{1}$ be defined by $f(z)=z^{2}$. If we choose $a=e^{i 2 \pi / 3}$, then $\rho(f, a)=2$. We have $\mathcal{R}\left(f^{n}\right)=\left\{R_{\theta}^{n} \mid \theta=\frac{3 k+1}{3 \cdot 2^{n}} 2 \pi, k=0,1, \ldots, 2^{n}-1\right\}$, where $R_{\theta}^{n}$ is an abbreviation for the notation $R_{e^{i \theta}}^{n}$ and every root class is a singleton (see [4, Chapter V.B. Example 3.3, p. 127]). Consider $n=3$, in this case, $R_{4 \pi / 3}^{3}$ is the center of $\mathcal{R}\left(f^{3}\right)$ and $R_{\pi / 12}^{(3)}=\left\{R_{\pi / 12}^{3}, R_{\pi / 3}^{3}, R_{4 \pi / 3}^{3}\right\}$, so $\ell\left(R_{\pi / 12}^{(3)}\right)=3$ and $\ell\left(R_{\pi / 3}^{(3)}\right)=2$. 
Let $w:(I, 0,1) \rightarrow(X, a, f(a))$ be a path, then there is a path $w^{(n)}$ : $(I, 0,1) \rightarrow\left(X, a, f^{n}(a)\right)$ defined by $w^{(n)}=w * f(w) * f^{2}(w) * \cdots * f^{n-1}(w)$ for $n>0$. We abbreviate the fundamental group $\pi_{1}(X, a)$ as $\pi$ and define a homomorphism $f_{w}^{n}: \pi \rightarrow \pi$ by $f_{w}^{n}([v])=\left[w^{(n)} * f^{n}(v) *\left(w^{(n)}\right)^{-1}\right]$. Note that $f_{w}^{n}=h_{w^{(n)}} \circ f_{\#}^{n}$, where $f_{\#}^{n}: \pi_{1}(X, a) \rightarrow \pi_{1}\left(X, f^{n}(a)\right)$ is the homomorphism induced by $f^{n}$ and $h_{w^{(n)}}$ is an isomorphism (see [5, Theorem 7.3.8, p. 384]). Let $f_{w}^{n}(\pi)=\phi^{(n)}$. Then there is an injective function $\theta_{n}: \mathcal{R}\left(f^{n}\right) \rightarrow \pi / \phi^{(n)}$ defined by

$$
\theta_{n}\left(R^{n}\right)=\phi^{(n)} \cdot\left[w^{(n)} * f^{n}(c)\right],
$$

where $c:(I, 0,1) \rightarrow(X, a, x)$ is a path for $x \in R^{n}$. The coset $\theta_{n}\left(R^{n}\right)$ in $\pi / \phi^{(n)}$ is called the coordinate of $R^{n}$ which is independent of the choice of the point $x \in R^{n}$ and of the path $c$ from $a$ to $x$ [1, Section 4].

We observe that $w^{(\rho)}$ is a loop at $a$ and $f^{\rho}$ induces $f_{\#}^{\rho}: \pi \rightarrow \pi$. Define a function $\left[f_{w}^{\rho}\right]: \pi / \phi^{(n)} \rightarrow \pi / \phi^{(n)}$ by

$$
\left[f_{w}^{\rho}\right]\left(\phi^{(n)} \cdot \gamma\right)=\phi^{(n)} \cdot f_{w}^{\rho}(\gamma) \cdot\left[w^{(\rho)}\right] .
$$

This is well defined because if $\phi^{(n)} \cdot \gamma=\phi^{(n)} \cdot \gamma^{\prime}$, then

$$
f_{w}^{\rho}\left(\gamma^{\prime}\right) \cdot\left[w^{(\rho)}\right] \cdot\left[w^{(\rho)}\right]^{-1} \cdot f_{w}^{\rho}\left(\gamma^{-1}\right)=f_{w}^{\rho}\left(\gamma^{\prime} \gamma^{-1}\right) \in f_{w}^{\rho}\left(\phi^{(n)}\right)=\phi^{(n+\rho)} \subseteq \phi^{(n)} .
$$

Thus we have the following algebraic version of Lemma 2.1.

Lemma 2.6. Suppose $\rho \leq n$ and $\phi^{(n)} \cdot \gamma \in \pi / \phi^{(n)}$, then there exist two-type sequences of elements of $\pi / \phi^{(n)}$ as follows:

(1): If $\rho \mid n$, then there exists a reduced sequence

$$
\phi^{(n)} \cdot \gamma,\left[f_{w}^{\rho}\right]\left(\phi^{(n)} \cdot \gamma\right), \ldots,\left[f_{w}^{\rho}\right]^{n / \rho}\left(\phi^{(n)} \cdot \gamma\right)=\phi^{(n)} \cdot\left[w^{(n)}\right] .
$$

(2): If $\rho \nmid n$, then there exists a reduced sequence

$$
\begin{aligned}
& \phi^{(n)} \cdot \gamma,\left[f_{w}^{\rho}\right]\left(\phi^{(n)} \cdot \gamma\right), \ldots,\left[f_{w}^{\rho}\right]^{m+1}\left(\phi^{(n)} \cdot \gamma\right)=\phi^{(n)} \cdot\left[w^{((m+1) \rho)}\right], \\
& \text { where } n=m \rho+r \text { and } 0<r<\rho \text { for some } m, r \in \mathbb{Z} .
\end{aligned}
$$

Proof. (1) If $\rho \mid n$, then there exists $m \in \mathbb{Z}$ such that $n / \rho=m$, so

$$
\begin{aligned}
{\left[f_{w}^{\rho}\right]^{m}\left(\phi^{(n)} \cdot \gamma\right) } & =\phi^{(n)} \cdot f_{w}^{m \rho}(\gamma) \cdot f_{w}^{(m-1) \rho}\left(\left[w^{(\rho)}\right]\right) \cdots \cdots f_{w}^{\rho}\left(\left[w^{(\rho)}\right]\right) \cdot\left[w^{(\rho)}\right] \\
& =\phi^{(n)} \cdot f_{w}^{(m-1) \rho}\left(\left[w^{(\rho)}\right]\right) \cdots \cdots f_{w}^{\rho}\left(\left[w^{(\rho)}\right]\right) \cdot\left[w^{(\rho)}\right] \\
& =\phi^{(n)} \cdot\left[w^{(n)}\right] .
\end{aligned}
$$

Furthermore, since $\left[f_{w}^{\rho}\right]\left(\phi^{(n)} \cdot\left[w^{(n)}\right]\right)=\phi^{(n)} \cdot\left[w^{(n+\rho)}\right]$ and $\left[w^{(n+\rho)}\right] \cdot\left[\left(w^{(n)}\right)^{-1}\right]=$ $f_{w}^{n}\left(\left[w^{(\rho)}\right]\right) \in \phi^{(n)}$, we have

$$
\left[f_{w}^{\rho}\right]^{m}\left(\phi^{(n)} \cdot \gamma\right)=\left[f_{w}^{\rho}\right]^{m+1}\left(\phi^{(n)} \cdot \gamma\right) .
$$

(2) If $\rho \nmid n$, then as $(1)$, we have $\left[f_{w}^{\rho}\right]^{m+1}\left(\phi^{(n)} \cdot \gamma\right)=\phi^{(n)} \cdot\left[w^{((m+1) \rho)}\right]$ and

$$
\left[w^{((m+2) \rho)}\right] \cdot\left[\left(w^{((m+1) \rho)}\right)^{-1}\right]=f_{w}^{(m+1) \rho}\left(w^{(\rho)}\right) \in \phi^{(m \rho+\rho)} \subseteq \phi^{(n)} .
$$

Thus we get $\left[f_{w}^{\rho}\right]^{m+1}\left(\phi^{(n)} \cdot \gamma\right)=\left[f_{w}^{\rho}\right]^{m+2}\left(\phi^{(n)} \cdot \gamma\right)$. 
In Lemma $2.6, \phi^{(n)} \cdot\left[w^{(n)}\right]$ and $\phi^{(n)} \cdot\left[w^{((m+1) \rho)}\right]$ play the role of the center of the set $\pi / \phi^{(n)}$ with respect to the recurrence number (compare Definition 2.2 above).

Definition 2.7. For $\rho \leq n$. The reduced $\left[f_{w}^{\rho}\right]$-orbit of the element $\phi^{(n)} \cdot \gamma$ of $\pi / \phi^{(n)}$ is the set

$$
\gamma^{(n)}=\left\{\phi^{(n)} \cdot \gamma,\left[f_{w}^{\rho}\right]\left(\phi^{(n)} \cdot \gamma\right), \ldots,\left[f_{w}^{\rho}\right]^{m}\left(\phi^{(n)} \cdot \gamma\right) \mid m \rho<n+\rho\right\} .
$$

We define the length $\ell\left(\gamma^{(n)}\right)$ of the reduced $\left[f_{w}^{\rho}\right]$-orbit $\gamma^{(n)}$ as $\ell\left(\gamma^{(n)}\right)=\# \gamma^{(n)}$ the cardinality of the set $\gamma^{(n)}$. The set of all such reduced $\left[f_{w}^{\rho}\right]$-orbits will be called the reduced $\left[f_{w}^{\rho}\right]$-orbit set of $f$, denoted by $\mathcal{R}_{\rho}^{(n)}\left(f_{w}\right)$.

Theorem 2.8. There is an injective function

$$
\theta_{n}: \mathcal{R}_{\rho}^{(n)}(f) \rightarrow \mathcal{R}_{\rho}^{(n)}\left(f_{w}\right)
$$

defined by $\theta_{n}\left(R^{(n)}\right)=\left[w^{(n)} * f^{n}(c)\right]^{(n)}$ for any path $c$ from a to $x$ in $R^{(n)}$, where $x$ in $R^{(n)}$ means that $x$ belongs to the first element of $R^{(n)}$.

Proof. By [1, Theorem 4.3], there is an injective function

$$
\theta_{n}: \mathcal{R}\left(f^{n}\right) \rightarrow \pi / \phi^{(n)}
$$

defined by $\theta_{n}\left(R^{n}\right)=\phi^{(n)} \cdot\left[w^{(n)} * f^{n}(c)\right]$ for any path $c$ from $a$ to $x$ in $R^{n}$.

We will show that under this injection $\theta_{n}$, the reduced $f^{\rho}$-orbit corresponds to the reduced $\left[f_{w}^{\rho}\right]$-orbit. That is to say, we show the commutativity of the following diagram:

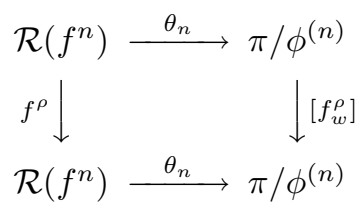

Then $\theta_{n}$ clearly induces the desired injection between the reduced orbit sets.

Let $R^{n} \in \mathcal{R}\left(f^{n}\right)$ and take a path $c:(I, 0,1) \rightarrow(X, a, x)$ for $x \in R^{n}$. Then the root $f^{\rho}(x)$ of $f^{n}$ is contained in the root class $f^{\rho}\left(R^{n}\right)$. Thus we have

$$
\begin{aligned}
{\left[f_{w}^{\rho}\right] \circ \theta_{n}\left(R^{n}\right) } & =\left[f_{w}^{\rho}\right]\left(\phi^{(n)} \cdot\left[w^{(n)} * f^{n}(c)\right]\right) \\
& =\phi^{(n)} \cdot f_{w}^{\rho}\left(\left[w^{(n)} * f^{n}(c)\right]\right) \cdot\left[w^{(\rho)}\right] \\
& =\phi^{(n)} \cdot\left[w^{(\rho)}\right] \cdot f_{\#}^{\rho}\left(\left[w^{(n)} * f^{n}(c)\right]\right) \\
& \left.=\phi^{(n)} \cdot\left[w^{(\rho)} * f^{\rho}\left(w^{(n)}\right) * f^{n+\rho}(c)\right]\right) \\
& =\phi^{(n)} \cdot\left[w^{(n)} * f^{n}\left(w^{(\rho)} * f^{\rho}(c)\right)\right] .
\end{aligned}
$$

On the other hand, $w^{(\rho)} * f^{\rho}(c)$ is a path from $a$ to $f^{\rho}(x)$ and the coordinate is independent of the choice of the path, then we have $\theta_{n} \circ f^{\rho}\left(R^{n}\right)=\theta_{n}\left(f^{\rho}\left(R^{n}\right)\right)=$ $\phi^{(n)} \cdot\left[w^{(n)} * f^{n}\left(w^{(\rho)} * f^{\rho}(c)\right)\right]$. 


\section{Full depth property of reduced orbits}

For $\rho<n$, let $R^{n-\rho} \in \mathcal{R}\left(f^{n-\rho}\right)$ and $x \in R^{n-\rho}$ be given. Define $i_{\rho, n}\left(R^{n-\rho}\right)$ as the unique root class of $\mathcal{R}\left(f^{n}\right)$ determined by $x$. This gives a function $i_{\rho, n}: \mathcal{R}\left(f^{n-\rho}\right) \rightarrow \mathcal{R}\left(f^{n}\right)$. Then

Theorem 3.1. [1] Let $f: X \rightarrow X$ be a selfmap of a path connected space and $\rho=\rho(f, a)<n$. Then the diagram

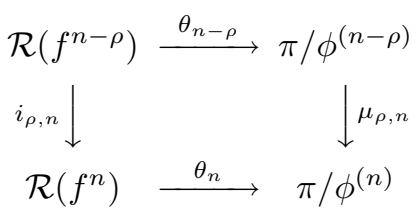

commutes, where $\mu_{\rho, n}$ is defined by $\mu_{\rho, n}\left(\phi^{(n-\rho)} \cdot \gamma\right)=\phi^{(n)} \cdot\left(\left[w^{(\rho)}\right] \cdot f_{\#}^{\rho}(\gamma)\right)$.

Proof. See [1, Theorem 4.5].

In [1, Section 4], if $\rho<\infty$, a root class $R^{n}$ of $f^{n}$ is reducible if $\rho<n$ and there exists $\gamma \in \pi$ such that $\theta_{n}\left(R^{n}\right)=\mu_{\rho, n}\left(\phi^{(n-\rho)} \cdot \gamma\right)$. Otherwise, it is irreducible. If $\rho=\infty$, then all the root classes of $f^{n}$ are irreducible. The reducibility of a root class is independent of the choice of $w$.

Combining Theorem 2.8 and Theorem 3.1 we have

Theorem 3.2. Let $f: X \rightarrow X$ be a selfmap of a path connected space and $\rho=\rho(f, a)<n$. Then the diagram of pointed sets

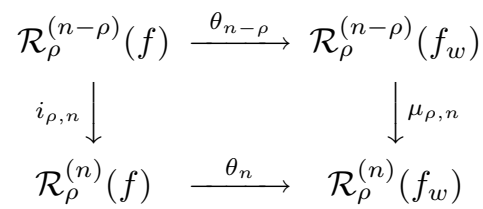

commutes, where $\mu_{\rho, n}\left(\gamma^{(n-\rho)}\right)=\left(\left[w^{(\rho)}\right] \cdot f_{\sharp}^{\rho}(\gamma)\right)^{(n)}$.

Moreover, if $\rho<k \rho<m \rho<n$, then $\mu_{m \rho, n}=\mu_{k \rho, n} \circ \mu_{(m-k) \rho, n-k \rho}$.

Definition 3.3. We say that a reduced $f^{\rho}$-orbit $R^{(n)}$ is reducible to level $n-m \rho$ if $\rho \leq m \rho<n$ and there exists $\gamma \in \pi$ such that $\theta_{n}\left(R^{(n)}\right)=\mu_{m \rho, n}\left(\gamma^{(n-m \rho)}\right)$ for some positive integer $m$. Otherwise, it is irreducible. The lowest level $d=d\left(R^{(n)}\right)$ to which $R^{(n)}$ reduces is its depth. A reduced $f^{\rho}$-orbit $R^{(n)}$ is said to have the full depth property if

$$
d\left(R^{(n)}\right)+\rho=\rho \cdot \ell\left(R^{(n)}\right)
$$

(compare [3, Definition 1.8]).

Remark 3.4. Note that in Theorem 3.2, if $\rho<m \rho<n$ and the reduced $f^{\rho}$-orbit of a root class is reducible to $n-m \rho$, then it is reducible to $n-\rho$. In other words, the reduced $f^{\rho}$-orbit of a root class is not reducible to $n-\rho$, then it is not reducible to $n-m \rho$. It follows that Definition 3.3 is well defined. 
Remark 3.5. Note that if $\rho \mid n$ and $\rho<n$, then the coordinate of the reduced $f^{\rho}$-orbit $R_{a}^{(n)}$ of the center $R_{a}^{n}$ is $\theta_{n}\left(R_{a}^{(n)}\right)=\left[w^{(n)}\right]^{(n)}=\mu_{n-\rho, n}\left(\left[w^{(\rho)}\right]^{(\rho)}\right)$ by Theorem 3.2, so $d\left(R_{a}^{(n)}\right)=\rho$. Thus it does not have the full depth property.

Theorem 3.6. Let $f: X \rightarrow X$ be a selfmap of a path connected space. If $\rho(f, a)=\rho \mid n$ and $R_{a}^{n} \in \mathcal{R}\left(f^{\rho}\right)$, then the reduced $f^{\rho}$-orbit of any root class of $f^{n}$ except the center has the full depth property.

Proof. Let $R^{(n)}$ be the reduced $f^{\rho}$-orbit of a root class $R^{n}$ and $n=k \rho$ for some $k \in \mathbb{Z}_{+}$. The commutativity of the diagram in Theorem 2.8 shows that the length of $R^{(n)}$ is equal to the length of its coordinate. If $\rho=n$, then clearly every reduced $f^{\rho}$-orbit except the center has the full depth property. Thus we can assume $\rho<n$.

Suppose that $\ell\left(R^{(n)}\right)=\ell>1$ and $\theta_{n}\left(R^{n}\right)=\phi^{(n)} \cdot\left[w^{(n)} * f^{n}(c)\right]$ for any path $c$ from $a$ to $x$ in $R^{n}$. Then, by the definition of length, we have

$$
\left[f_{w}^{\rho}\right]^{\ell-1}\left(\theta_{n}\left(R^{n}\right)\right)=\phi^{(n)} \cdot w^{(n)}=\theta_{n}\left(R_{a}^{n}\right)
$$

and

$$
\left[f_{w}^{\rho}\right]^{\ell-1}\left(\theta_{n}\left(R^{n}\right)\right)=\phi^{(n)} \cdot\left[w^{(n)} * f^{n}\left(w^{((\ell-1) \rho)} * f^{(\ell-1) \rho}(c)\right)\right] .
$$

Since $f^{(\ell-1) \rho}(x) \in f^{(\ell-1) \rho}\left(R^{n}\right)=R_{a}^{n}$ and $R_{a}^{n} \in \mathcal{R}\left(f^{\rho}\right)$ by hypothesis, there exists a path $p: I \rightarrow X$ from $f^{(\ell-1) \rho}(x)$ to $a$ such that $f^{\rho} \circ p \simeq a_{*}$. For the loop $w^{((\ell-1) \rho)} * f^{(\ell-1) \rho}(c) * p$ at $a$ and $\rho \leq n-(\ell-1) \rho<n$, we have

$$
\begin{aligned}
& \mu_{n-(\ell-1) \rho, n}\left(\phi^{((\ell-1) \rho)} \cdot\left(w^{((\ell-1) \rho)} * f^{(\ell-1) \rho}(c) * p\right)\right. \\
= & \phi^{(n)} \cdot\left(\left[w^{(n-(\ell-1) \rho)}\right] \cdot f_{\sharp}^{n-(\ell-1) \rho}\left(w^{((\ell-1) \rho)} * f^{(\ell-1) \rho}(c) * p\right)\right. \\
= & \phi^{(n)} \cdot w^{(n)} \cdot f^{n}(c) \cdot f^{n-(\ell-1) \rho}(p) \\
= & \phi^{(n)} \cdot w^{(n)} \cdot f^{n}(c) \\
= & \theta_{n}\left(R^{n}\right) .
\end{aligned}
$$

Therefore we have $d \leq(\ell-1) \rho$ by the definition of depth.

On the other hand, suppose that $d\left(R^{(n)}\right)=d=k \rho-m \rho>0$. Then, by the definition of depth, there exists $\gamma \in \pi$ such that $\theta_{n}\left(R^{n}\right)=\mu_{m \rho, n}\left(\phi^{(n-m \rho)}\right.$. $\gamma)$. By the commutativity of the diagram in Theorem 3.2 and Lemma 2.6(1), $\left[f_{w}^{\rho}\right]^{k-m}\left(\phi^{((k-m) \rho)} \cdot \gamma\right)=\phi^{((k-m) \rho)} \cdot w^{((k-m) \rho)}$, this implies that

$$
\begin{aligned}
{\left[f_{w}^{\rho}\right]^{k-m}\left(\theta_{n}\left(R^{n}\right)\right) } & =\left[f_{w}^{\rho}\right]^{k-m} \circ \mu_{m \rho, n}\left(\phi^{(n-m \rho)} \cdot \gamma\right) \\
& =\mu_{m \rho, n} \circ\left[f_{w}^{\rho}\right]^{k-m}\left(\phi^{(n-m \rho)} \cdot \gamma\right) \\
& =\mu_{m \rho, n}\left(\phi^{((k-m) \rho)} \cdot w^{((k-m) \rho)}\right) \\
& =\phi^{(n)} \cdot\left(\left[w^{(m \rho)}\right] \cdot f_{\sharp}^{m \rho}\left(w^{((k-m) \rho)}\right)\right) \\
& =\phi^{(n)} \cdot w^{(n)} .
\end{aligned}
$$

This means that $\ell-1 \leq k-m$ by the definition of length, so $(\ell-1) \rho \leq$ $(k-m) \rho=d$. 
Example 3.7. As in [1, Example 6.1], let $S^{1}=\{z:|z|=1\}$ be the unit circle in the complex plane and let $f: S^{1} \rightarrow S^{1}$ be defined by $f(z)=z^{3}$. If we choose $a=1$, then $\rho(f, a)=1$. A direct computation shows that $\mathcal{R}\left(f^{n}\right)=$ $\left\{z_{k}^{n}=e^{\frac{k}{3^{n}} 2 \pi i} \mid k=0,1, \ldots, 3^{n}-1\right\}$, where every root class is a singleton. Taking $w$ be the constant path and $c_{k}$ the arc from 1 to $z_{k}^{n}$ in the counterclockwise direction, the coordinate $\theta_{n}\left(z_{k}^{n}\right)$ of the root class $z_{k}^{n}$ can be identified with $k \in \pi / \phi^{(n)}=\mathbb{Z}_{3^{n}}$. Since $f_{\sharp}: \pi \rightarrow \pi$ is given by $f_{\sharp}(\gamma)=3 \gamma$ and $\mu_{m, n}$ is determined by $f_{\sharp}^{m}$ for $m<n$, it follows that the reduced $f$-orbit $z_{k}^{(n)}$ of the root class $z_{k}^{n}$ is reducible to $n-m$ if and only if $k \equiv 0\left(\bmod 3^{m}\right)$. By Theorem 3.6, every reduced $f$-orbit except the center has the full depth property.

If we choose $a=e^{\pi i / 2}$, then $\rho(f, a)=2$. Thus we have $\mathcal{R}\left(f^{n}\right)=\left\{z_{k}^{n}=\right.$ $\left.e^{\frac{4 k+1}{4 \cdot 3^{n}} 2 \pi i} \mid k=0,1, \ldots, 3^{n}-1\right\}$. Taking $w$ be the arc from $a$ to $f(a)$ in the counter-clockwise direction and $c_{k}$ the arc from $a$ to $z_{k}^{n}$ in the counter-clockwise direction, as the above case, the coordinate $\theta_{n}\left(z_{k}^{n}\right)$ of the root class $z_{k}^{n}$ can be identified with $k \in \pi / \phi^{(n)}=\mathbb{Z}_{3^{n}}$. Consider $n=3$, in this case, $\mu_{2,3}$ is determined by $w^{(2)}+f_{\sharp}^{2}$, it follows that the reduced $f^{\rho}$-orbit $z_{k}^{(3)}$ of the root class $z_{k}^{3}$ is reducible to 1 if and only if $k-2 \equiv 0\left(\bmod 3^{2}\right)$. Thus we have $d\left(z_{11}^{(3)}\right)=1$ and $\ell\left(z_{11}^{(3)}\right)=2$, so $z_{11}^{(3)}$ does not have the full depth property.

\section{References}

[1] R. F. Brown, B. Jiang and H. Schirmer, Roots of iterates of maps, Topology Appl. 66 (1995), 129-157.

[2] B. Jiang, Lectures on Nielsen Fixed Point Theory, Contemporary Mathematics 14 American Mathematical Society, Providence, RI, 1983.

[3] B. Jiang, S. H. Lee, and M. H. Woo, Reidemeister orbit sets, Fund. Math. 183 (2004), $139-156$.

[4] T. Kiang, The Theory of Fixed Point Classes, Springer, Berlin, 1989.

[5] E. Spanier, Algebraic Topology, McGraw-Hill, New York, 1966.

Department of Mathematics

MOKWON UNIVERSITY

DAEJEON 302-318, KOREA

E-mail address: seoungho@mokwon.ac.kr 